ARTICLE OPEN

\title{
Large positive correlation between the effective electron mass and the multipolar fluctuation in the heavy-fermion metal
}

$\mathrm{Ce}_{1-x} \mathrm{La}_{x} \mathrm{~B}_{6}$

\author{
Dongjin Jang $\mathbb{D}^{1,2}$, Pavlo Y. Portnichenko ${ }^{3}$, Alistair S. Cameron ${ }^{3}$, Gerd Friemel ${ }^{4}$, Anatoliy V. Dukhnenko ${ }^{5}$, Natalya Y. Shitsevalova ${ }^{5}$, \\ Volodymyr B. Filipov ${ }^{5}$, Astrid Schneidewind $\mathbb{1 0}^{6}$, Alexandre Ivanov ${ }^{7}$, Dmytro S. Inosov (iD ${ }^{3}$ and Manuel Brando ${ }^{1}$
}

For the last few decades, researchers have been intrigued by multipolar ordering phenomena and related quantum phase transitions in heavy-fermion Kondo systems. However, a criticality induced by substitution level $(x)$, temperature $(T)$, or magnetic field $(B)$ is poorly understood even in the prototypical material, $C_{1_{-x}} L_{x} B_{6}$, despite a large collection of experimental results is available. In this work, we present $T-B, x-T$, and $x-B$ phase diagrams of $C \mathrm{e}_{1-x} \mathrm{La}_{x} \mathrm{~B}_{6}$ (B $\|$ [110]). These are completed by investigating heat capacity, magnetocaloric effect (MCE), and elastic neutron scattering. A drastic increase of the Sommerfeld coefficient $\gamma_{0}$, which is estimated from the heat capacity down to $0.05 \mathrm{~K}$, is observed with increasing $x$. The precise $T-B$ phase diagram including a high-entropy region is derived from the MCE analysis in which a knowledge beyond the equilibrium thermodynamics is involved. Finally, the $x-B$ phase diagram at $T=0$, which supports the existence of a quantum critical point at $x>0.75$, is obtained by the same analysis. A detailed interpretation of phase diagrams strongly indicates positive correlation between the fluctuating multipoles and the effective electron mass.

npj Quantum Materials (2017)2:62 ; doi:10.1038/s41535-017-0068-x

\section{INTRODUCTION}

The identification of multipolar moments from the physical properties of $f$-electron systems has been one of the prime subjects in condensed matter physics. A quadrupolar ordering has been reported from representative materials such as $\mathrm{CeB}_{6}$ (ref. 1), $\mathrm{URu}_{2} \mathrm{Si}_{2}$ (refs. 2-4), $\mathrm{UPd}_{3}$ (refs. 5, 6), $\mathrm{PrPd}_{3}$ (ref. 7), $\mathrm{Ce}_{3} \mathrm{Pd}_{20} \mathrm{Ge}_{6}$ (ref. 8), $\operatorname{Pr}_{3} \mathrm{Pd}_{20} \mathrm{Si}_{6}$ (ref. 9), $\operatorname{PrTi}_{2} \mathrm{Al}_{20}$ (ref. 10), $\operatorname{PrV}_{2} \mathrm{Al}_{20}$ (refs. 11, 12), and $\mathrm{YbRu}_{2} \mathrm{Ge}_{2}$ (ref. 13). An octupolar moment is suggested as the major order parameter in $\mathrm{NpO}_{2}$ (refs. 14-16), while the octupoles order under very high magnetic field above $35 \mathrm{~T}$ in $\mathrm{URu}_{2} \mathrm{Si}_{2}$ (ref. 2). Except these materials, $\mathrm{Ce}_{1-x} \mathrm{La}_{x} \mathrm{~B}_{6}$ deserves a special interest as it is possible to control the appearance of different multipolar phases in the ground state (GS) by varying the substitution level $(x) .^{17-19}$ In certain ranges of $x$, temperature $(T)$, and external magnetic field (B), antiferromagnetic (AFM), ${ }^{20-22}$ antiferroquadrupolar (AFQ), ${ }^{1,23-25}$ and antiferro-octupolar (AFO) ${ }^{26-30}$ phases are all observable. Therefore, $\mathrm{Ce}_{1-x} \mathrm{La}_{x} \mathrm{~B}_{6}$ can be regarded as a model system to study rather general features of multipolar phases and superexchange interactions between multipolar moments. ${ }^{31-33}$ Furthermore, recent discoveries of superconductivity (SC) near quadrupolar quantum critical point $(\mathrm{QCP})^{10,11}$ strongly motivate researchers to investigate one of unresolved problems about the relation between the substitution-tuned multipolar QCP and proximate competing order parameters, including SC that occurs on the La-rich side in $\mathrm{Ce}_{1-x} \mathrm{La}_{x} \mathrm{~B}_{6}{ }^{34}$ By convention for naming primary order parameters, the spin/pseudospin (orbital) paramagnetic (PM) phase, the AFQ phase, the AFM phase, and the AFO phase are referred to as phases I, II, III, and IV, respectively.

On the other hand, even though largely scattered information about the multipolar phase transitions is gathered, ${ }^{35}$ the result is rather difficult to understand. The $x-T$ phase diagram at $B=0$ reveals a weakening of the phase IV as $T$ is increased, while the $x-$ $B$ phase diagram at $T=0$ reveals an enhancement of the same phase as $B$ is increased. Therefore, a discontinuity of the phase IV has been introduced along the $x$-axis in the up to date $x-T-B$ phase diagram of $\mathrm{Ce}_{1-x} \mathrm{La}_{x} \mathrm{~B}_{6}$, and the quantum criticality at the verge of the phase IV is poorly understood.

In this article, we investigate physical properties of $\mathrm{Ce}_{1-x} \mathrm{La}_{x} \mathrm{~B}_{6}$ $(x=0,0.18,0.23,0.28,0.5,0.75)$ by measuring heat capacity at ambient pressure, magnetic Bragg intensity, and the magnetocaloric effect (MCE) with B $\|$ [110]. $C_{p}$ and elastic neutron scattering (NS) measurements are analyzed to construct $x$-dependent $T-B$ phase diagrams and the $x-T$ phase diagram which show intricate transitions between multipolar phases. The analysis of MCE in this system suggests not only more precise $T-B$ phase diagrams but also an existence of an exotic high-entropy region. Meanwhile, a discovery of a highly irreversible MCE across a magnetic phase boundary is in contradiction to the widely accepted description of the MCE. From the low- $T$ analysis of $C_{p} / T$, the Sommerfeld coefficient, $\gamma_{0}$, is extracted, and $\gamma_{0}(B)$ exhibits local maxima at phase boundaries in $x-B$ plane. Finally, we present an understandable $x-T$ and $x-B$ phase diagrams, which imply an

\footnotetext{
${ }^{1}$ Max Planck Institut für Chemische Physik fester Stoffe, 01187 Dresden, Germany; ${ }^{2}$ Max Planck-POSTECH/KOREA Center for Complex Phase Materials, 77 Cheongam-Ro, Pohang, Republic of Korea; ${ }^{3}$ Institut für Festkörper- und Materialphysik, TU Dresden, 01069 Dresden, Germany; ${ }^{4}$ Max Planck Institut für Festköperforschung, 70569 Stuttgart, Germany; ${ }^{5}$ I. M. Frantsevich Institute for Problems of Materials Science of NAS, 3 Krzhyzhanovsky Street, Kiev 03680, Ukraine; ${ }^{6}$ Jülich Center for Neutron Science (JCNS), Forschungszentrum

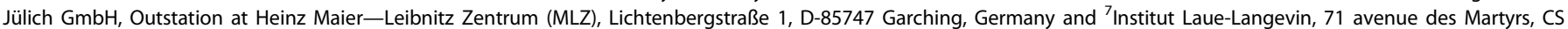
20156, 38042 Grenoble cedex 9, France

Correspondence: Dongjin Jang (dongjin.jang@cpfs.mpg.de)
}

Received: 26 June 2017 Revised: 12 October 2017 Accepted: 17 October 2017

Published online: 10 November 2017 
emergence of a putative QCP as phase IV is suppressed. The observations concerning the variation of $y_{0}$ on the $x-B$ plane are explicable by a large positive correlation between multipolar fluctuations and the effective electron mass, $m^{*}$, within heavy Fermi-liquid (FL) description. This simple conclusion seems generic and valid in aforementioned multipolar heavy fermion metals.

\section{RESULTS}

In Fig. $1 \mathrm{a}$, phase transitions in $\mathrm{Ce}_{1-x} \mathrm{La}_{x} \mathrm{~B}_{6}$ are signaled by sharp anomalies in $C_{p} / T$ if the Ce concentration is high. As $x$ is increased, the anomalies are weakened and the overall magnitude of $C_{p}(T) / T$ is diminished. We remind that according to previous NS experiments, $^{36}$ substitution of La for Ce leads to an electronic doping effect, yet no additional broadening of the quasielastic line is observed due to impurity scattering. Therefore, the influence of La as an impurity can be considered negligible. To define the critical temperature, it should be reminded that the mean-field (MF) theory predicts a discontinuous jump in $C_{p} / T$ at the transition temperature. We can extrapolate the discontinuous jump as noted by the red and blue lines drawn in Fig. 1b. Here, the so-called method of entropy balance is applied to define the Néel temperature $T_{\mathrm{N}}$ and the AFQ transition temperature $T_{\mathrm{Q}}$ : Note that hatched regions with the same color have the same area. On the other hand, a transition from a non-collinear AFM phase (III) to a collinear AFM phase (III') at $T_{N}^{\prime}$ typically results in a kink in $C_{p} / T$. Each region of multipolar phases and the corresponding transitions are confirmed from the literature. ${ }^{35,37}$ The field is applied along [110] crystallographic orientation because a brief $x-T-B$ phase diagram is only available for this direction as a refs. $35,37$. Figure 1c shows that the entropy as a function of $T$ loses its features for the phase transitions (see kinks in the black curve at $T_{\mathrm{N}}=2.4 \mathrm{~K}$ and $T_{\mathrm{Q}}=3.3 \mathrm{~K}$ ) as $x$ is increased. The overall magnitude of the entropy per 1 mole of $\mathrm{Ce}$ is increased with $x$. Although $\Gamma_{8}$ quartet split from the 6-fold degenerate wave functions of an isolated $\mathrm{Ce}^{3+}$ ion is known be the $\mathrm{GS}^{38}$ the saturation entropy ( $R \ln 4)$ of the non-interacting quartets is observed well above $10 \mathrm{~K}$ (ref. 39) due to the remaining short-range multipolar interactions.

Figure 2 shows the field-induced Bragg intensity at the $(1 / 21 / 21 / 2)$ wave vector in three samples of $\mathrm{Ce}_{1-x} \mathrm{La}_{x} \mathrm{~B}_{6}$ measured by NS. In addition, field-dependent AFM Bragg intensities have also been measured and analyzed (not shown, see ref. 37). Unlike the AFM phase, ${ }^{21,22}$ which exhibits Bragg scattering in zero magnetic field, the hidden-order phase II can only be visualized by the elastic intensity that originates from the field-induced dipolar moments modulated by the underlying AFQ structure. Not only does this offer a method to reveal the presence of AFQ correlations by NS, but it may also provide information about the type of the multipoles involved. ${ }^{40}$

The field dependence in Fig. 2 reveals that the onset of intensity measured at $(1 / 21 / 21 / 2)$ in all the studied samples occurs in several steps. In $\mathrm{Ce}_{0.82} \mathrm{La}_{0.18} \mathrm{~B}_{6}$ (Fig. 2a), the intensity remains almost zero within the phase III, but starts to increase already at $B$ $=1.5 \mathrm{~T}$ inside the phase III' for $T=0.07 \mathrm{~K}$. This demonstrates that this distinct phase represents a combination of conventional (dipolar) AFM and AFQ order parameters, in contrast to the purely dipolar phase III. Upon entering the phase II, an even steeper jump in intensity is observed around 2.2 T. With increasing temperature, both transitions are shifted towards lower fields as the AFM phases are suppressed. A similar two-step increase without fieldhistory dependence is also seen in the low-temperature measurement on $\mathrm{Ce}_{0.77} \mathrm{La}_{0.23} \mathrm{~B}_{6}$ (Fig. 2b). In our third sample, $\mathrm{Ce}_{0.72} \mathrm{La}_{0.28} \mathrm{~B}_{6}$, the zero-field GS is reportedly taken over by the phase IV (refs. 18, 19), but the phases III and III' can still be stabilized by the application of field. As a result, the corresponding field dependence in Fig. 2c now exhibits a series of three steps at $T$ $=0.1 \mathrm{~K}$ : a broadened transition near $0.5 \mathrm{~T}$ from phase IV to III (more clearly seen as the onset of AFM Bragg intensity, see ref. 37), a kink around $1.5 \mathrm{~T}$ corresponding to the III-III' transition, and the third transition to phase II at $2.5 \mathrm{~T}$ (ref. 19). We note that for the La substitution level of $x=0.28$, some initial increase in field-induced intensity is observed already within phase III, unlike at low La concentrations where no signal within phase III was found. This suggests that the field-stabilized phase III in La-substituted samples is different from the zero-field phase III with regard to its intermixing with the AFQ order parameter. On the other hand, within phase IV, i.e., for fields below $1 \mathrm{~T}$, the (1/2 1/2 1/2) intensity is only weakly sensitive to the field.

$T-B$ phase diagrams of $\mathrm{Ce}_{1-x} \mathrm{La}_{x} \mathrm{~B}_{6}$ with $\mathbf{B} \|$ [110] are displayed in Fig. 3. Phase boundaries and the degree of critical fluctuations are readily recognizable by the contrasting colors in the contour plots of $C_{p}(T, B) / T$. The low- $T$ phase boundaries (yellow solid lines) are determined from the MCE analysis which will be explained with Fig. 4. The notations for the critical fields are straightforward, e.g., the critical field across the phases I and II is labeled by $B_{I-\mid I}$. In Fig. $3 \mathrm{~b}-\mathrm{d}$, the red upward-triangle and the red downward-triangle denote $B_{\mathrm{II}-\mathrm{II}{ }^{\prime}}$ and $B_{\mathrm{III-III}}$ determined by NS shown in Fig. 2. In phase II, the field-induced AFO order stabilizes the AFQ order resulting in
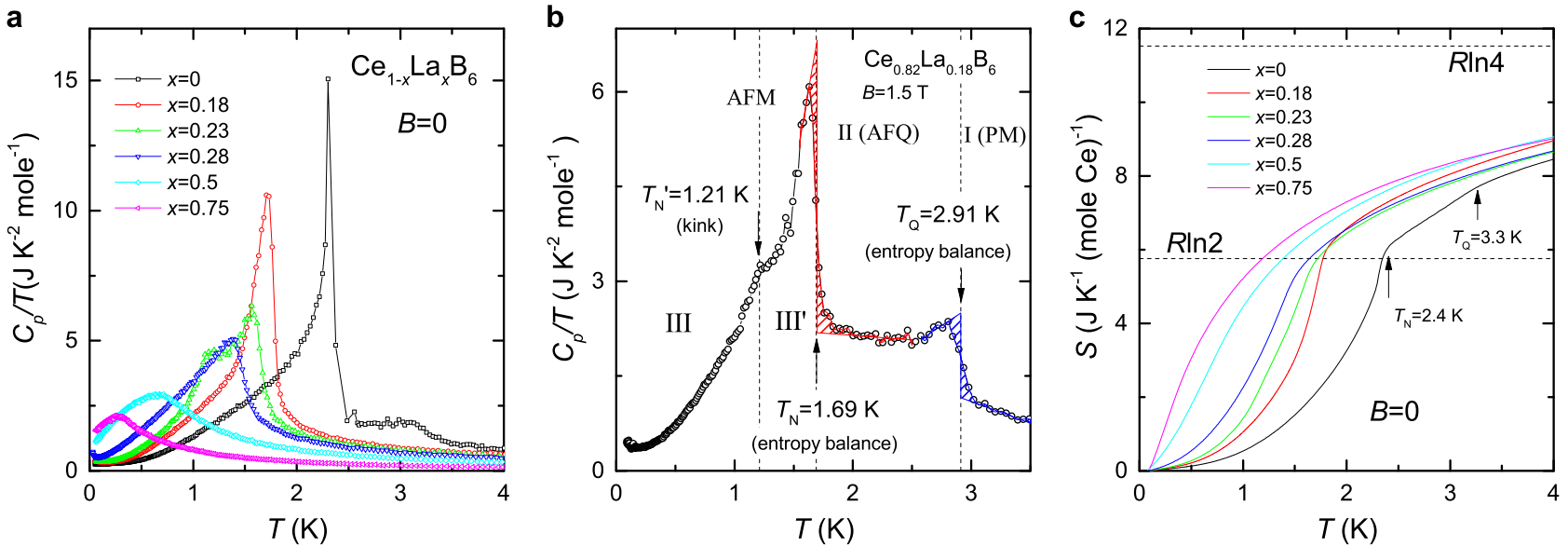

Fig. 1 Specific heat capacity $C_{p}(T)$ and entropy $S_{p}(T)$ with $x=0,0.18,0.230 .28,0.5,0.75$. a $T$-dependent specific heat functions with different

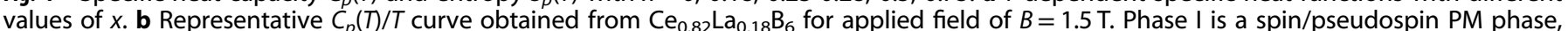
phase II is AFQ phase, phase III is a non-colinear AFM phase, and phase III' is a collinear AFM phase. To find the MF transition temperature, we used method of entropy balance resulting in $T_{\mathrm{N}}=1.69 \mathrm{~K}$ (red line and red hatched area) and $T_{\mathrm{Q}}=2.91 \mathrm{~K}$ (blue line and blue hatched area). The transition between AFM phases is indicated by the kink at $T_{\mathrm{N}}^{\prime}=1.21 \mathrm{~K}$. c Entropy curves per 1 mole of Ce atom are exhibited for samples of different values of $x$ 

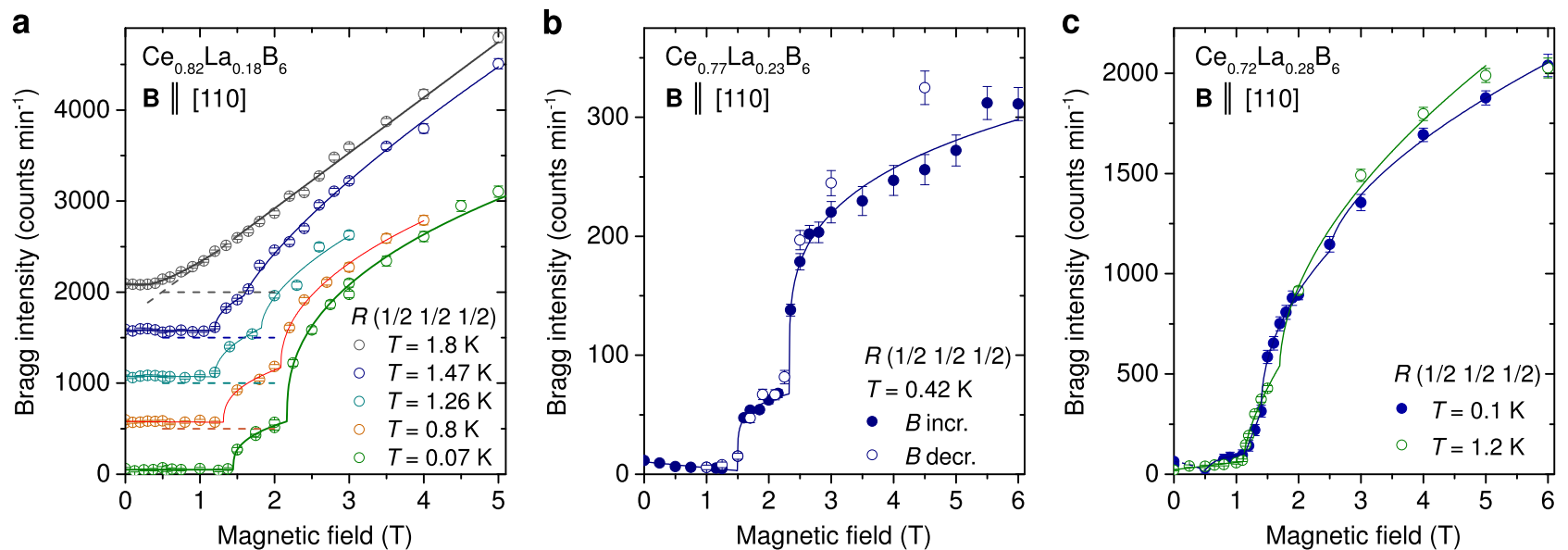

Fig. $2 B$-dependent and T-dependent (1/2 1/2 1/2) Bragg intensity from $\mathrm{Ce}_{1-x} \mathrm{La}_{x} \mathrm{~B}_{6}$ with $x=0.18,0.23$, and 0.28 . a Field-induced (1/2 1/2 1/2) Bragg intensity measured from $\mathrm{Ce}_{0.82} \mathrm{La}_{0.18} \mathrm{~B}_{6}$ with different temperatures are shown. Each curve is shifted along the $y$ axis for increased visibility. The lowest lying curve is for the lowest $T$. b Field-induced (1/2 1/2 1/2) Bragg intensity from Ce $\mathrm{C}_{0.77} \mathrm{La}_{0.23} \mathrm{~B}_{6}$ with $T=0.42 \mathrm{~K}$. Filled circles designate neutron counts in increasing $B$. Hollow circles designate neutron counts in decreasing B. c Field-induced (1/2 1/2 1/2) Bragg intensity obtained from $\mathrm{Ce}_{0.72} \mathrm{La}_{0.28} \mathrm{~B}_{6}$ at two different temperatures. Filled circles and hollow circles denote the neutron counts at $T=0.1 \mathrm{~K}$ and $T=1.2 \mathrm{~K}$, respectively. B $\|[110]$ in all of the measurements

positive slope of $B_{\mathrm{I}-\mathrm{II}}(T){ }^{29}$ Phase II is gradually weakened and transformed into phase I with increasing $x$.

For $x=0.23$, a certain phase appears in the temperature range of $1 \mathrm{~K}<T<1.6 \mathrm{~K}$ and fields below $1 \mathrm{~T}$ (Fig. $3 \mathrm{C}$ ). When $x=0.28$, this phase becomes the GS (Fig. 3d). Since the primary order parameter of this phase has not been conclusively determined, it is customarily called phase IV. The nature of phase IV will be discussed later. In Fig. 3e, broad peaks are almost insensitive to fields below $2 \mathrm{~T}$. It will be argued that the phase IV survives in $\mathrm{Ce}_{0.5} \mathrm{La}_{0.5} \mathrm{~B}_{6}$ with a rather broad heat capacity anomaly. In Fig. $3 \mathrm{f}$, $\mathrm{Ce}_{0.25} \mathrm{La}_{0.75} \mathrm{~B}_{6}$ shows field-insensitive peaks only in the low- $T$ and low- $B$ region. In the rest of the $T-B$ plane, a specific-heat anomaly is broadened and moves to the high- $T$ side as $B$ is increased. This is reminiscent of the Kondo impurity behavior, but the detailed shape of $C_{p}(T, B) / T$ deviates from the resonance-level model. ${ }^{41,42}$

Now, we present the quasi-adiabatic MCE observed in $\mathrm{Ce}_{1-x} \mathrm{La}_{x} \mathrm{~B}_{6}$. The ideal MCE is defined as reversible temperature changes of a thermally isolated specimen controlled by an external magnetic field. Suppose the $S_{p, B_{\mathrm{i}}}(T)$ curve is lying higher than the $S_{p, B_{\mathrm{f}}}(T)$ curve. The sample tends to reduce its entropy by releasing a certain amount of heat as the field is changed from $B_{\mathrm{i}}$ to $B_{\mathrm{f}}$. But the heat must be dissipated and the temperature has to be increased to keep the entropy unchanged. In the reverse procedure, cooling of the sample occurs. Likewise, a phase transition from a magnetically disordered state to an ordered state is described by a sharp increase of the sample temperature and vice versa. In practice, however, the sample is in a quasi-adiabatic condition in which the sample temperature, $T_{\text {qad, }}$ slowly and monotonically relaxes to $T_{\text {mix }}$ the temperature of the mixing chamber of the dilution fridge. Moreover, a field-induced heating around a critical region is found to be essential to describe the irreversible $T_{\text {qad }}(B)$ upon field cycling. We have constructed a mathematical model (see Supplementary Note 2) for the observed MCE, and the basic principle is graphically summarized in Fig. 4. Eddy current heating is negligible in this experiment (see Supplementary Note 2).

The background of Fig. 4a represents the color-coded entropy $S_{p}(T, B)$ of $\mathrm{CeB}_{6}$ calculated from $C_{p} / T$. Black (red) solid lines indicate $T_{\text {qad }}(B)$ when the field is increased (decreased). In Fig. 4b, representative magnetocaloric (MC) sweeps with $T_{\text {mix }}=0.2 \mathrm{~K}$ and the sweep-rate $r=0.1 \mathrm{~T} \mathrm{~min}^{-1}$ are magnified. To simulate $T_{\text {qad }}(B)$, we assume a continuous phase transition, and start with a reversible ansatz for the true MCE. The distribution of the excessive field-induced heat, $d Q(B) / d B$, is also approximated in terms of an analytic function. Then, these factors are taken into the differential equation for the theoretical quasi-adiabatic temperature and consecutively adjusted until the solution is optimized to $T_{\text {qad }}(B)$. Figure $4 \mathrm{c}$ is the amplified view of Fig. $4 \mathrm{~b}$ at low- $B$. Here, the reversible ansatz is the blue solid line. In Fig. $4 d$, the envelope of the black hatched-area (red-area) pertains to $d Q$ $(B) / d B$ in sweep-up (sweep-down) mode. The optimized solutions are denoted by dash-dotted lines in Fig. 4c. Note that the major cause of the irreversibility is indeed the field-induced heating accompanied by the maximum MCE. Magnified view of $T_{\text {qad }}(B)$ near the III-III' boundary is shown in Fig. 4e. About two times larger change of $T_{\text {qad }}(B)$ in down-sweep is well reproduced by the hysteretic field-induced heating (Fig. 4e, f). The II-III' boundary is also explained by the model (not shown). We tried to simulate the total field-induced heat, $Q$, as closely as possible for both sweep directions, but no constraint regarding $d Q / d B$ was imposed. These conditions reflect hysteretic behaviors across certain phase boundaries observed in magnetic susceptibility, magnetization, and NS. ${ }^{18,37}$

The yellow solid lines in Fig. 4a, g, m connect critical points referenced to steepest slopes in the reversible ansatzes (see blue vertical dashed lines in other panels of the Fig. 4). On the other hand, a line which fades away above $T=0.4 \mathrm{~K}$ is inserted in Fig. $4 \mathrm{a}$ and stresses that the MCE is weakened as the temperature is increased. The shape of the reversible ansatz confirms that the magnetic entropy under this line is larger than the magnetic entropy of the well-defined AFM phase. While this unclosed borderline cannot be attributed to a phase transition in the strict sense, AFM domain selection or motion would also be insufficient to explain the origin of this line as these effects usually continue to $T_{\mathrm{N}}$ (ref. 18).

Figure $4 \mathrm{~g}$ shows the $B-T$ phase diagram of $\mathrm{Ce}_{0.77} \mathrm{La}_{0.23} \mathrm{~B}_{6}$. Curves of $T_{\text {qad }}(B)$ with $T_{\text {mix }}=0.4 \mathrm{~K}$ and $r=0.04 \mathrm{~T} \mathrm{~min}^{-1}$ are magnified in Fig. $4 \mathrm{~h}$. The model is applied to explain the weak features around $B=0.4 \mathrm{~T}$ (Fig. $4 \mathrm{i}, \mathrm{j}$ ). The phase II-III' boundary is also analyzed (Fig. $4 \mathrm{k}, \mathrm{I}$ ). The unclosed borderline with the color gradient is shifted to higher fields compared with the one found in $\mathrm{CeB}_{6}$.

In Fig. $4 \mathrm{~m}$, phase IV becomes the GS. The model is applied to the representative $T_{\text {qad }}(B)$ shown in Fig. $4 \mathrm{n}$. Analyses of III-IV, III-III' (not shown), and II-III' transitions repeatedly confirm that substantial $Q$ is accompanied by the large MCE (Fig. 4o-r). The III-IV boundary found by the MCE analysis is distinct, but we 

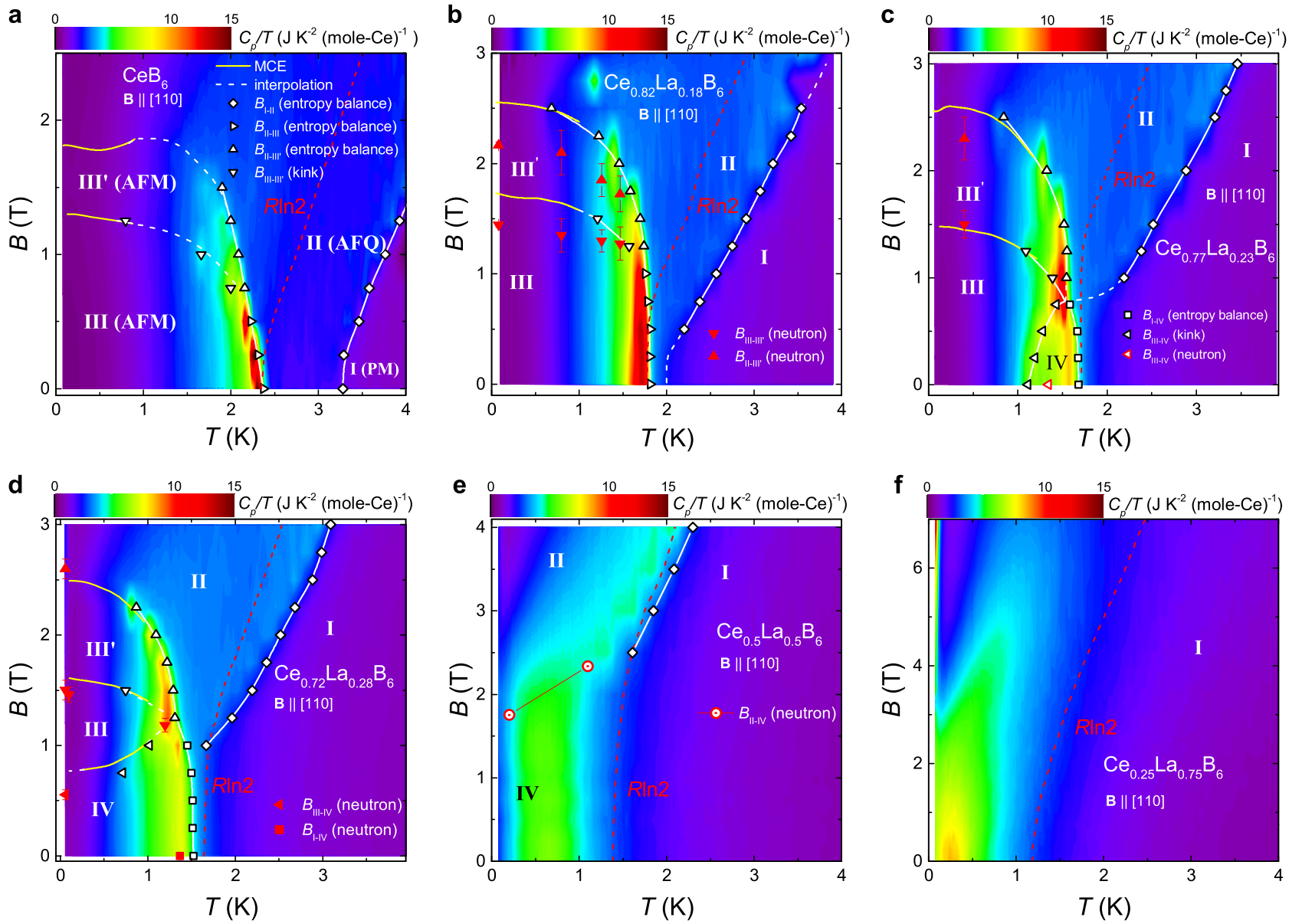

Fig. $3 T-B$ phase diagrams of $C_{e_{1-x}} L_{x} B_{6}$ with $\mathbf{B} \|$ [110]. a The color plot is interpolated from $C_{p, B}(T) / T$ of $C e B_{6}$. Transition temperatures are determined by an entropy balance or by a notable kink in $C_{p, B}(T) / T$. In the $T-B$ plane, critical fields are estimated as a function of $T$ (see the legend). $\mathbf{b}$ The color plot is interpolated from $C_{p, B}(T) / T$ of $\mathrm{Ce}_{0.82} \mathrm{La}_{0.18} \mathrm{~B}_{6}$. The critical fields determined from the changes in the $(1 / 21 / 21 / 2)$ Bragg intensity are superimposed. Upward triangles in red denotes the II-III transition and downwards triangles in red denotes the III-III' transition. c When $x=0.23$, phase II exists only if a field is applied and phase IV appears in a narrow region. Transitions between phase I and phase IV found by entropy balance are marked by white squares. White left-triangles denote transitions between phase III and phase IV. The left-triangle with red-edge marks a change of Bragg intensity when the system enters to phase III from phase IV. d For $x=0.28$, phase IV becomes the GS and the red square notes that there is a change in magnetic Bragg intensity. The left-triangle in red denotes $B_{\mathrm{III}-\mathrm{IV}}$ taken from Fig. 2c. e When $x=0.5$, phase III is absent and a broad phase transition between phases I and IV is remained. (1/2 1/2 1/2) Bragg intensity is induced by field upon entering phase II from phase IV (see dotted-circles with red edge). $f$ As $x$ is increased to 0.75 , we cannot find a notable sign for a distinct phase transition except rather sharp features at the lower-left corner. Red dashed-lines in all of the panels represent isentropic lines with $S_{p}=R \ln 2$

cannot draw such a well-defined phase boundary by observing a low- $T$ AFM structure since it is strongly field-history-dependent. ${ }^{37}$ Hence, the sudden onset of the AFQ phase with field-induced Bragg intensity (see Fig. 2c) might explain the sharp III-IV boundary.

\section{DISCUSSION}

Before we discuss our new findings, it has to be emphasized that the MCE analysis has played an essential role to present new perspectives on thermodynamic phenomena in $\mathrm{Ce}_{1-x} \mathrm{La}_{x} \mathrm{~B}_{6}$. Experimentally, the completion of the precise $B-T$ phase diagram is possible because $T_{\text {qad }}(B)$ is extremely sensitive to the entropy change, and this property allows us to explore relevant and the most interesting area in the $B-T$ plane where anomalies in $C_{p}$, NS, ultrasonic attenuation, ${ }^{43}$ and thermal expansion ${ }^{44}$ are not easily detectable. Theoretically, our phenomenological model implies substantial $r$-dependent quantum mechanical friction appears due to strongly fluctuating local magnetic moments, and suggest a non-equilibrium formulation for a field-driven phase transition. In terms of a quasiparticle dynamics, it is suspected that inelastic magnon-magnon scatterings might be prominent. On the belief that the above observation could be universal in systems showing field-driven phase transition, a detailed study about a $r$-dependent $Q$ and a type of transition is now underway: A first-order phase transition is expected if both $Q$ and $d Q / d B$ are highly irreversible as in the III-IV transition (Fig. 4p), while a second-order transition is characterized by reversible $Q$ and $d Q / d B$ (Fig. $4 \mathrm{l}, \mathrm{q}$ ). A continuous III-III' transition is described by reversible $Q$ and irreversible $d Q / d B$ because of the domain hysteresis (Fig. $4 \mathrm{f}$ ).

In $\mathrm{CeB}_{6}$, we observe $S_{p}=R \ln 2$ exactly at $T_{N}$ (Fig. 3a). This indicates that, below $T_{N}$, the two lowest-lying energy levels are approximately $k_{\mathrm{B}} T_{\mathrm{N}}$ apart from each other while, above $T_{\mathrm{N}}$, the level scheme transforms into two separate Kramers doublets suitable for the AFQ ordering. In $\mathrm{Ce}_{0.82} \mathrm{La}_{0.18} \mathrm{~B}_{6}$, the AFQ phase is weakened and the AFM phase is confined by a vertical phase boundary at low- $B$ (Fig. $3 b$ ). The isentropic line with $S_{p}=R \ln 2$ follows this vertical boundary validating the above mentioned fundamental change in the energy level scheme. 

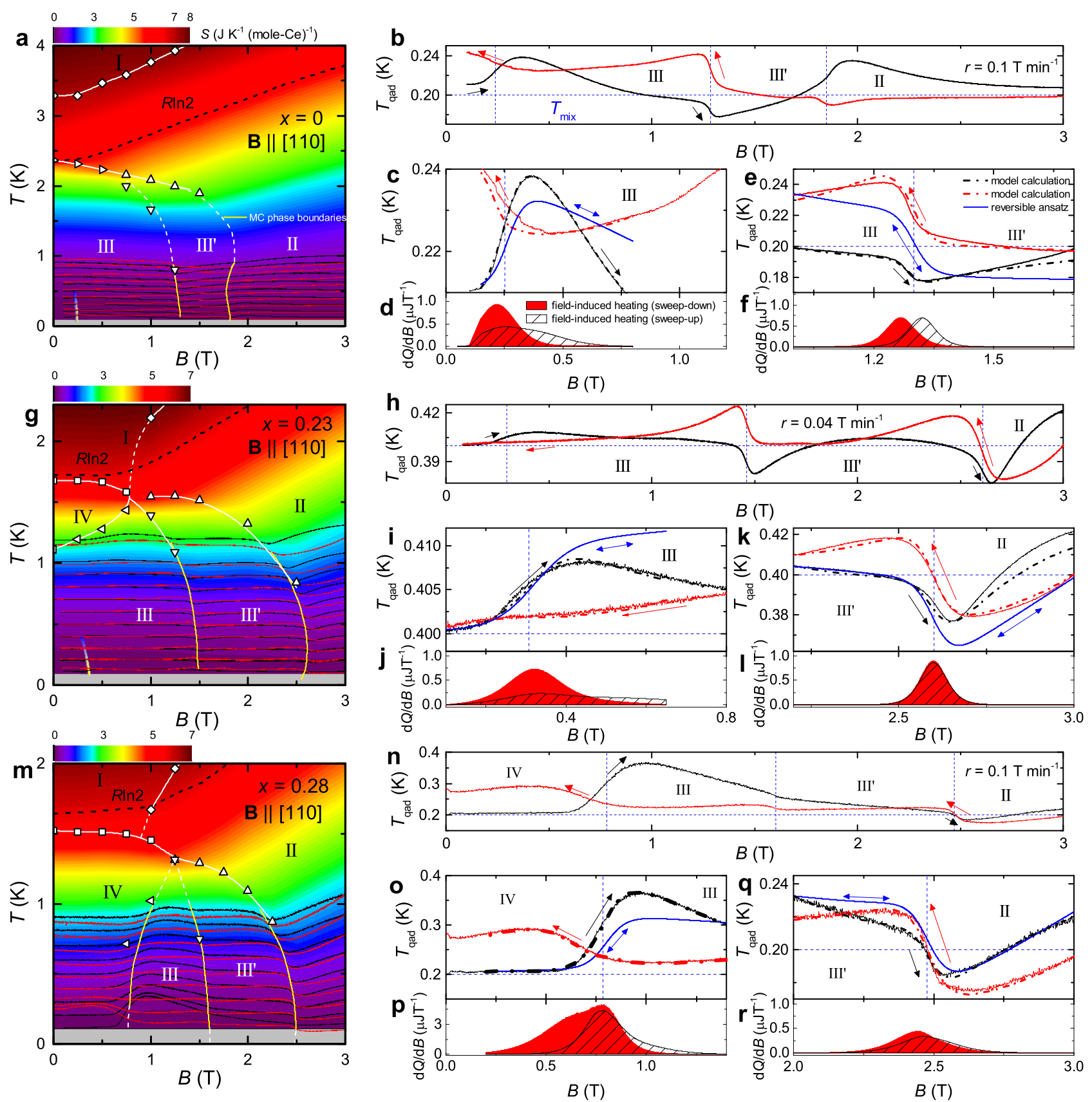

Fig. $4 S_{p}(B, T), B-T$ phase diagrams, and quasi-adiabatic $M C$ anomalies in $C_{1-x} L_{a} B_{6}$ with applied fields parallel to [110] direction. a The background is the contour plot of $S_{p}(B, T)$ from $\mathrm{CeB}_{6}$. Yellow solid lines denote phase boundaries determined by $M C$ anomalies. Black (red) line is $T_{\text {qad }}(B)$ in up-(down-) sweep with sweep-rate $r=0.1 \mathrm{~T} \mathrm{~min}^{-1}$. Near the lower-left corner unclosed borderline under which the entropy is higher than that of phase III is emphasized by the color gradient. b One of the $T_{\text {qad }}(B)$ curves is magnified from $\mathbf{a}$. $T_{\text {mix }}$ is fixed at $0.2 \mathrm{~K}$. c $T_{\text {qad }}(B)$ curves below 1.2 T are enlarged from $\mathbf{b}$. The blue solid line is the reversible ansatz. The black and red dash-dotted lines are calculated by the numerical model explained in the text and the Supplementary Note 2. $\mathbf{d}$ The envelopes of the black-hatched and the red-filled areas denote distributions of the field-induced heat $d Q(B) / d B$ in sweep-up and down, respectively. e Region around the III-III' boundary is magnified from b. Results of the model calculations and the reversible ansatz are shown together. $\mathbf{f} d Q(B) / d B$ curves around the III-III' boundary are shown. $\mathbf{g}$ Contour plot of $S_{p}(B, T)$ and the $B-T$ phase diagram of $C_{0.77} L_{0.23} B_{6}$ are exhibited with $r=0.04 \mathrm{~T} \mathrm{~min}^{-1}$. As in a, the high-entropy region is noted by the unclosed borderline with color gradient. $\mathbf{h}$ Curves for $T_{\mathrm{qad}}(B)$ with $T_{\text {mix }}=0.4 \mathrm{~K}$ are magnified from $\mathbf{g}$. $\mathbf{i}-\mathbf{I} \mathrm{MC}$ anomalies for selected boundaries from the $\mathbf{h}$ are analyzed. $\mathbf{m}$ Contour plot of $S_{p}(B, T)$ and the $B-T$ phase diagram of $C_{0.72} \operatorname{La}_{0.28} B_{6}$ are shown. $r=0.1 \mathrm{~T}$ min ${ }^{-1}$. $\mathbf{n} T_{\text {qad }}(B)$ curves with $T_{\text {mix }}=0.2 \mathrm{~K}$ are magnified from $\mathbf{m}$. o-r $\mathrm{MC}$ anomalies around selected phase boundaries from $\mathbf{n}$ are analyzed

The phase IV appears in $\mathrm{Ce}_{0.77} \mathrm{La}_{0.23} \mathrm{~B}_{6}$ and it is stabilized in $\mathrm{Ce}_{0.72} \mathrm{La}_{0.28} \mathrm{~B}_{6}$ with sharp $C_{p}$ anomalies indicating long-range ordering (Fig. 3c, d). The I-IV boundary is almost vertical and the isentropic line with $S_{p}=R \ln 2$ is following this phase boundary. While up to date resonant X-ray scattering experiment, ${ }^{27,29,30} \mathrm{NS}^{28}$ suggest the primary order parameter is of AFO type with the AFQ and the AFM moments as field-induced secondary orders, the vertical I-IV boundary indicate that the lowest-lying levels in phase IV are barely affected by the Zeeman effect. Even if an isolated CEF GS is the $\Gamma_{8}$ quartet, exchange interactions between 
a

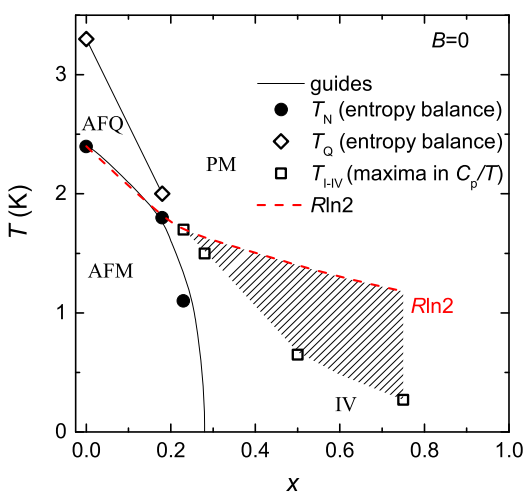

b

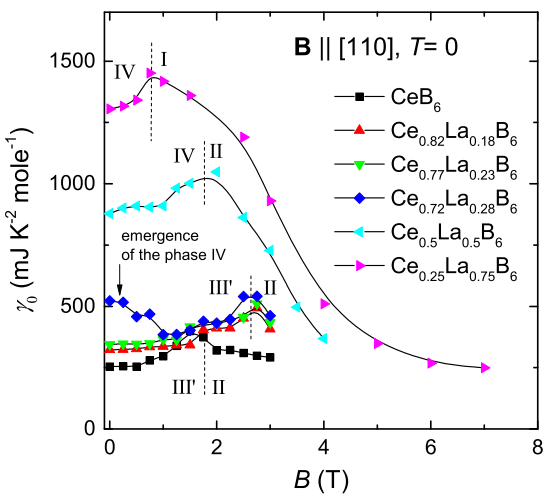

C

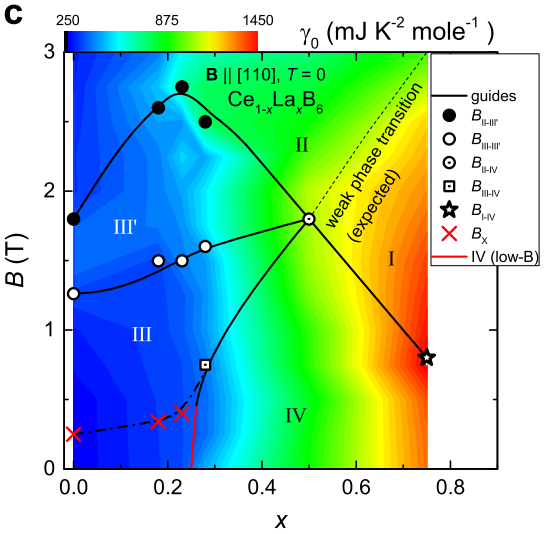

Fig. 5 The $B=0 x-T$ phase diagram, $B$-dependent and $x$-dependent $\gamma_{0}$, and $T=0 x-B$ phase diagram of $C e_{1-x} L_{x} B_{6}$. a $B=0 x-T$ phase diagram is shown. It is completed by placing coordinates of specific heat anomalies in the $x-T$ plane. The I-IV transition with transition temperature $T_{1-I V}$ becomes broad above $x \simeq 0.3$. The region of broad transitions is roughly coincident with the hatched area between the $S_{p}=R \ln 2$ isentropic line and the $T_{1-I V}(x)$. $\mathbf{b}$ The Sommerfeld coefficient as a function of $B$ with different values of $x$ is presented. Locations of local maxima (see short vertical dashed lines) are coincident with the critical points extrapolated from the lowest- $T$ MCE anomalies. c The black dot and the white circle designate $B_{\|-I I I^{\prime}}$ and $B_{\text {III-III' }}$ determined by the MCE analysis, respectively. The high-entropy region exists below $B_{\times}$and the black dashdotted line connects $\times$ symbols as a guide to the eye. The red segment for the low- $B$ III-IV phase boundary is to emphasizes large increase of $\gamma_{0}$ upon entering the phase IV which can be confirmed in $\mathbf{b}$. $B_{\text {IIIIV }}$ (white dotted-circle), and $B_{\text {I-IV }}$ (white star) are also determined by the MCE analysis (see Supplementary Figs. 2 and 3, and Supplementary Note 3). The background is contour plot of $\gamma_{0}(x, B)$

15 possible order parameters could further lift the degeneracy of wave functions at each Ce-site. In reality, at $T_{1-I V}$, the level scheme seems to change into two doublets, and $S_{p}=R \ln 2$ along the vertical section of the I-IV boundary originates from the lower doublet. We also suggest that, when major exchange terms are taken into account, an effective $g$-factor with respect to the lowest lying doublet is very small at low- $B$.

In $\mathrm{Ce}_{0.5} \mathrm{La}_{0.5} \mathrm{~B}_{6}$, sharp $C_{p}$ anomalies are absent, but the above proposed properties of phase IV seem to persist below $2 \mathrm{~T}$ (Fig. 3e and Supplementary Fig. 1). The phase IV is easily suppressed and transformed into phase $\mathrm{I}$ in the region where $B>1 \mathrm{~T}$ and $T>0.25 \mathrm{~K}$ for $\mathrm{Ce}_{0.25} \mathrm{La}_{0.75} \mathrm{~B}_{6}$ (see Fig. $3 f$ and Supplementary Fig. 3).

Figure 5 a shows that energy scales of AF-exchange interactions between multipolar moments are so close that most of the multipolar order parameters are competing in the limited region of the $x-T$ space. The temperature difference between the point where the entropy of $R \ln 2$ is released and the point where the peak in $C_{p} / T$ is observed (hollow square) provides an estimate for the range of critical fluctuations. As noted by the hatched area, the MF-like $C_{p}$ anomalies are smoothed towards the putative QCP above $x=0.75$.

In Fig. $5 b, Y_{0}(x, B)$ is extracted from $C_{p}(T, B) / T$ by estimating constant contribution at low-T. For $x=0$, the FL behavior is obvious and the constant value of $C_{p}(T, B) / T$ below $0.5 \mathrm{~K}$ (Fig. 1 ) is taken as $\gamma_{0}$. As $x$ is increased, the temperature range for the FL behavior diminishes, but we can easily extract $y_{0}(x, B)$. Even though clear FL behavior is not seen down to the lowest temperature as in phase IV $(x>0.28)$, we can still extrapolate $\gamma_{0}$ because $C_{p}(T, B) / T$ shows linear $T$-dependence below $0.2 \mathrm{~K}$. An applied field suppresses this $T$-linear dependence, and clear $F L$ behavior with a part of the nuclear Schottky contribution $\left(\propto 1 / T^{3}\right)$ is observed in $C_{p}(T, B) / T$. In this case, we simply subtract $1 / T^{3}$ term to get $\gamma_{0}$. Curves of $\gamma_{0, x}(B)$ have local maxima at critical points as marked by the vertical dashed lines. Also, the magnitude of $\gamma_{0}$ drastically and monotonically increases with $x$ inside phase IV. Previous resistivity measurements also exhibit a similar increase in $m^{*}$ but there was no specific tendency with $x$ (ref. 45).

Symbols in Fig. 5c denote critical fields determined by the MCE analysis with $T_{\text {mix }}=0.02 \mathrm{~K}$ and the background is the contour plot of $\gamma_{0}(x, B)$. Representative $T_{\text {qad }}(B)$ curves with $x=0.5$ and $x=0.75$ are shown in the Supplementary Figs. 2 and 3. Compared to the latest $x-B$ phase diagram which is completed by collecting largely scattered experimental results, ${ }^{35}$ the current $x-B$ phase diagram is more reliable in three aspects. First, critical fields are well matched with the values of the peak positions in $\gamma_{0, x}(B)$ (Fig. 5b). Second, as $x$ is increased above 0.5 , phase IV boundary shows negative slope. Then the discontinuity in the previously reported $x-B$ phase diagram due to the misguided positive slope of phase IV boundary is eliminated (ref. 19). Although it is not well defined, SC emerges as $x$ approaches to 1 . This confirms the extinction of phase IV and the existence of a putative QCP in the range of $0.75<x<1$. Perhaps, the maximum $\gamma_{0}$ will be observed at the QCP as expected from Fig. 5b, c. Thirdly, a weak phase transition between phase I and phase II is suggested (see dashed line in upper-right area of Fig. 5c) which is consistent with the observation from Fig. $3 e, f$ that phase I will occupy the region in phase space for composition $x=$ 0.75 where phase II existed for $x=0.5$ (see Fig. 3, Supplementary Fig. 3 and Supplementary Note 3 for more details).

The essence of our study is that not only substantial modifications for the long-believed $x-B$ phase diagram of $\mathrm{Ce}_{1-x} \mathrm{La}_{x} \mathrm{~B}_{6}$ are made but also a clear indication of a positive correlation between multipolar fluctuations and $m^{*}$ is found. The increasing tendency of $\gamma_{0}$ along $x$ appears with increased multipolar fluctuations due to reduced and distributed superexchange interactions. In general, multipolar fluctuations in a certain phase imply fluctuations of the primary order parameter and field-induced order parameters. For instance, we have to consider fluctuating AFO order with field induced AFM and AFQ fluctuations in phase IV. To discuss $B$-dependence of $\gamma_{0}$, we remind that the external field suppresses multipolar fluctuations and aligns spins. However, given that fluctuations of order parameters are pronounced upon phase transitions, an observation of the maximum $\gamma_{0, x}(B)$ on a phase boundary definitely suggests the positive correlation between multipolar fluctuations and $\mathrm{m}^{*}$. Earlier result of Nakamura et al. ${ }^{45}$ shows small peaks of $A$ coefficient $\left(\alpha \mathrm{m}^{*}\right)$ on II-III boundary with B \|| [001], and partially supports our observation. When $x=0.5$, the peak of $\gamma_{0, x}(B)$ centered at $B=1.8 \mathrm{~T}$ is rather broad (Fig. $5 \mathrm{~b}$, left triangles). We deduce that the critical area around $x=0.5$ and $B=1.8 \mathrm{~T}$ (Fig. $5 \mathrm{C}$ ) is rather wide because fluctuations of order parameters in five different phases are focused.

By examining $\mathrm{Ce}_{1-x} \mathrm{La}_{x} \mathrm{~B}_{6}$ which exhibits almost all kinds of $\mathrm{AF}$ multipolar phase transition, we have drawn the simple conclusion that $m^{*}$ is positively correlated with order parameter fluctuations 
on an arbitrary phase boundary. We expect this phenomenon would be universal among less complicated systems mentioned in the introduction, and it is strongly suggested to reinvestigate thermodynamics of multipolar heavy fermion metals. Besides, the extensive field-sweep dependent heating across a phase boundary is observed and analyzed (Fig. 4). Although MCE was crucial to reveal AFO hidden order in $\mathrm{URh}_{2} \mathrm{Si}_{2}$ (ref. 2), the non-equilibrium contribution has not been reported probably due to rather fast relaxation of heat to the thermal reservoir which makes it very difficult to observe the non-equilibrium heating. Therefore, extensive examination of any material showing a field-driven phase transition with the method introduced in this article might bring far-reaching consequence which alters general equilibrium description of MCE.

As to the high-entropy region below $B_{\times}$in Fig. $5 c$, we speculate that a thermally unstable portion of heavy electrons (as described by the unclosed lines in Fig. $4 \mathrm{a}, \mathrm{g}$ ) is segregated from the system. Such a phase segregation would induce more quasiparticle degrees of freedom below the black dash-dotted line in Fig. $5 \mathrm{c}$ and the entropy will be increased. With $B=0, \gamma_{0}$ is doubled compared to its value at $x=0$ as the red solid line in Fig. $5 c$ is crossed from the left (see also the annotated curve connecting diamond symbols in Fig. 5b). This sudden increase of $\gamma_{0}$ can be triggered if itinerant electrons which might have been segregated to form the high-entropy region are participating in the correlation phenomenon relevant to $m^{*}$ in phase IV.

In the near future, we hope experimental techniques such as scanning tunneling spectroscopy, ${ }^{46}$ nonresonant inelastic X-ray scattering ${ }^{4}$ and inelastic $\mathrm{NS}^{47}$ are accessible for the further characterization of both the heavy itinerant electronic state and the multipolar state in $\mathrm{Ce}_{1-x} \mathrm{La}_{x} \mathrm{~B}_{6}$. Also, high-quality single crystals with $0.75<x<1$ will allow us to scrutinize the existence of a putative QCP and its possible conjunction with the SC which emerges as $x$ approaches to unity. ${ }^{34}$ We expect an identification of the role of multipolar fluctuations in forming SC will greatly expand our knowledge regarding multipolar QCP beyond what we know from recently discovered SC emerging from pressuretuned quadrupolar QCP in $\operatorname{PrTi}_{2} \mathrm{Al}_{20}{ }^{10}$ and field-induced quadrupolar QCP in $\operatorname{PrV}_{2} \mathrm{Al}_{20}{ }^{12}$

To conclude, a set of temperature $(T)$ - external field $(B)$ phase diagrams of $\mathrm{Ce}_{1-x} \mathrm{La}_{x} \mathrm{~B}_{6}$ is completed by analyzing specific heat capacity, elastic NS, and the MCE. The analysis of the MCE rectifies the previous $x-B$ phase diagram at $T=0$, and reveals a highentropy region in lower-left corner of the modified phase diagram. In contrast to the conventional reversible description of the MCE, a strong irreversibility is discovered across a magnetic phase boundary. It appears that considerable amount of heat is released from scattering between competing order parameters, and we believe the same could be observed in various other systems showing field-driven magnetic transitions. The $x-T$ phase diagram reveals competitions between multipolar energy scales at temperatures below $4 \mathrm{~K}$. Local maxima of the Sommerfeld coefficient $\gamma_{0}$ are developed on phase boundaries while overall magnitude of $\gamma_{0}$ increases with $x$. The systematic change of $\gamma_{0}(x, B)$ on the $x-B$ phase diagram explicitly indicates a large positive correlation between fluctuating multipoles and the effective electron mass in the heavy FL state of $\mathrm{Ce}_{1-x} \mathrm{La}_{x} \mathrm{~B}_{6}$.

\section{METHODS}

Material preparation and characterization

Single crystalline $\mathrm{Ce}_{1-x} \mathrm{La}_{x} \mathrm{~B}_{6}$ has been prepared by the floating-zone method as described elsewhere. ${ }^{48}$ For thermodynamic measurements, we used smaller pieces of the same single crystals that were used in earlier neutron-scattering studies. ${ }^{36}$ The structure belongs to the $P m \overline{3} m$ space group and the lattice constant is $4.14 \AA$ for $\mathrm{CeB}_{6}$. The orientation of the specimen was determined by X-ray Laue backscattering.
Heat capacity

The specific heat capacity is measured by using a compensated heat pulse method. ${ }^{49} \mathrm{~A}$ reliable measurement is made in the temperature range from 0.05 to $4 \mathrm{~K}$, and the external magnetic field has been applied up to $4 \mathrm{~T}$ in the ${ }^{3} \mathrm{He} /{ }^{4} \mathrm{He}$ dilution-fridge with $14 \mathrm{~T}$ magnet (Oxford Instrument).

\section{Elastic NS}

Elastic NS measurements were performed using the cold-neutron triple-axis spectrometers IN14 at the ILL, Grenoble (Fig. 2a, c) and the JCNS instrument PANDA $^{50}$ at FRM-II, Garching (Fig. 2b). The samples were mounted in a standard cryomagnet with the magnetic field applied along the [110] direction of the crystal. Measurements were performed using the conventional triple-axis configuration, with a cold beryllium filter on the diffracted beam to filter out higher-order contamination. The total intensity of the Bragg peak was determined by integrating the intensity either along a longitudinal scan through the $R(1 / 21 / 21 / 2)$ point, performed for Fig. $2 \mathrm{a}$, or through the integration of a rocking scan through the same point in Fig. $2 b, c$.

\section{MC effect}

Quasi-adiabatic condition were created by supporting the sample holder with thin nylon wires. The relaxation of the sample temperature to the temperature of the mixing chamber of the dilution-fridge is well described by a heat transfer equation and the relaxation time is about $4 \mathrm{~h}$ from $1 \mathrm{~K}$ Therefore, a sweep-rate has been chosen so that the total period of a fieldsweep is shorter than $4 \mathrm{~h}$. Under the above conditions, the sample temperature is monitored as a function of the external magnetic field.

Data availability

The original data which provide a foundation of the current article are available from the authors.

\section{ACKNOWLEDGEMENTS}

We appreciate fruitful discussions with P. Thalmeier, S. Wirth and S. Gönnenwein. This work is funded by the Max Planck-POSTECH Center for Complex Phase Materials KR2011-0031558 and by the German Research Foundation (DFG) within the Research Unit 960 Quantum Phase Transitions, individual Grant No. IN 209/3-1 and the Research Training Group GRK 1621

\section{AUTHOR CONTRIBUTIONS}

D.S.I. and M.B. instigated this work. A.V.D., N.Y.S, and V.B.F synthesized single crystals. P.Y.P., A.S.C., G.F. and D.S.I. performed elastic neutron scattering and interpreted the result. A.S. and A.I. provided instrument support during NS measurements. D.J.J. measured the heat capacity and the magnetocaloric effect. D.J.J. devised the analytic model for the quasi-adiabatic MCE. D.J.J. and D.S.I. wrote the manuscript. All of the authors participated in the discussion to interpret the results.

\section{ADDITIONAL INFORMATION}

Supplementary information accompanies the paper on the npj Quantum Materials website (https://doi.org/10.1038/s41535-017-0068-x).

Competing interests: The authors declare that they have no competing financial interests.

Publisher's note: Springer Nature remains neutral with regard to jurisdictional claims in published maps and institutional affiliations.

\section{REFERENCES}

1. Schenck, A., Gygax, F. N. \& Kunii, S. Field-induced magnetization distribution and antiferroquadrupolar order in $\mathrm{CeB}_{6}$. Phys. Rev. Lett. 89, 037201 (2002).

2. Jaime, M., Kim, K. H., Jorge, G., McCall, S. \& Mydosh, J. A. High magnetic field studies of the hidden order transition in $\mathrm{URu}_{2} \mathrm{Si}_{2}$. Phys. Rev. Lett. 89, 287201 (2002).

3. Elgazzar, S., Rusz, J., Amft, M., Oppeneer, P. M. \& Mydosh, J. A. Hidden order in $\mathrm{URu}_{2} \mathrm{Si}_{2}$ originates from Fermi surface gapping induced by dynamic symmetry breaking. Nat. Mater. 8, 337-341 (2009).

4. Sundermanna, M. et al. Direct bulk-sensitive probe of $5 f$ symmetry in $U R u_{2} \mathrm{Si}_{2}$. Proc. Natl. Acad. Sci. USA 113, 13989 (2016). 
5. McMorrow, D. F., McEwen, K. A., Steigenberger, U., Rønnow, H. M. \& Yakhou, F. XRay resonant scattering study of the quadrupolar order in $\mathrm{UPd}_{3}$. Phys. Rev. Lett. 87, 057201 (2001).

6. Walker, H. C. et al. Determination of the antiferroquadrupolar order parameters in $\mathrm{UPd}_{3}$. Phys. Rev. Lett. 97, 137203 (2006).

7. Onimaru, T. et al. Observation of modulated quadrupolar structures in $\mathrm{PrPb}_{3}$. Phys. Rev. Lett. 94, 197201 (2005).

8. Kitagawa, J., Takeda, N. \& Ishikawa, M. Possible quadrupolar ordering in a Kondolattice compound $\mathrm{Ce}_{3} \mathrm{Pd}_{20} \mathrm{Ge}_{6}$. Phys. Rev. B 53, 5101-5103 (1996).

9. Steinke, L. et al. Role of hyperfine coupling in magnetic and quadrupolar ordering of $\mathrm{Pr}_{3} \mathrm{Pd}_{20} \mathrm{Si}_{6}$. Phys. Rev. Lett. 111, 077202 (2013).

10. Matsubayashi, K. et al. Pressure-induced heavy fermion superconductivity in the nonmagnetic quadrupolar system $\mathrm{PrTi}_{2} \mathrm{Al}_{20}$. Phys. Rev. Lett. 109, 187004 (2012).

11. Tsujimoto, M., Matsumoto, Y., Tomita, T., Sakai, A. \& Nakatsuji, S. Heavy-fermion superconductivity in the quadrupole ordered state of $\operatorname{PrV}_{2} \mathrm{Al}_{20}$. Phys. Rev. Lett. 113, 267001 (2014).

12. Shimura, Y. et al. Field-induced quadrupolar quantum criticality in $\operatorname{PrV}_{2} \mathrm{Al}_{20}$. Phys. Rev. B 91, 241102(R) (2015).

13. Takimoto, T. \& Thalmeier, P. Theory of induced quadrupolar order in tetragonal $\mathrm{YbRu}_{2} \mathrm{Ge}_{2}$. Phys. Rev. B 77, 045105 (2008).

14. Santini, P. \& Amoretti, G. Magnetic-octupole order in neptunium dioxide? Phys. Rev. Lett. 85, 2188-2191 (2000).

15. Santini, P., Carreta, S., Magnani, N., Amoretti, G. \& Caciuffo, R. Hidden order and low-energy excitations in $\mathrm{NpO}_{2}$. Phys. Rev. Lett. 97, 207203 (2006).

16. Vitova, T. et al. The role of the $5 f$ valence orbitals of early actinides in chemical bonding. Nat. Commun. 8, 16053 (2017).

17. Hiroi, M., Kobayashi, S., Sera, M., Kobayashi, N. \& Kunii, S. Unusual magnetic phase diagram of $\mathrm{Ce}_{x} \mathrm{La}_{1-x} \mathrm{~B}_{6}(x=0.5$ 0.75). J. Phys. Soc. Jpn. 66, 1762-1770 (1997).

18. Tayama, T., Sakakibara, T., Tenya, K., Amitsuka, H. \& Kunii, S. Magnetic phase diagram of $\mathrm{Ce}_{x} \mathrm{La}_{1-x} \mathrm{~B}_{6}$ studied by static magnetization measurement at very low temperatures. J. Phys. Soc. Jpn. 66, 2268-2271 (1997).

19. Kobayashi, S., Sera, M., Hiroi, M., Kobayashi, N. \& Kunii, S. Transport properties in phase IV of $\mathrm{Ce}_{x} \mathrm{La}_{1-x} \mathrm{~B}_{6}$. J. Phys. Soc. Jpn. 69, 926-936 (2000).

20. Takigawa, M., Yasuoka, H., Tanaka, T. \& Ishizawa, Y. NMR study on the spin structure of $\mathrm{CeB}_{6}$. J. Phys. Soc. Jpn. 52, 728-731 (1983).

21. Effantin, J. M. et al. Magnetic phase diagram of $\mathrm{CeB}_{6}$. J. Magn. Magn. Mater. 47, 48, 145-148 (1985).

22. Zaharko, O. et al. Zero-field magnetic structure in $\mathrm{CeB}_{6}$ reinvestigated by neutron diffraction and muon spin relaxation. Phys. Rev. B 68, 214401 (2003).

23. Sakai, O., Shiina, R., Shiba, H. \& Thalmeier, P. A new interpretation of NMR in quadrupolar ordering phase of $\mathrm{CeB}_{6}$ - consistency with neutron scattering. $J$. Phys. Soc. Jpn. 66, 3005-3007 (1997).

24. Nakao, $\mathrm{H}$. et al. Antiferro-quadrupole ordering of $\mathrm{CeB}_{6}$ studied by resonant X-Ray scattering. J. Phys. Soc. Jpn. 70, 1857-1860 (2001).

25. Sera, M. et al. Anomalous temperature dependence of the magnetic field induced antiferromagnetic moment in the antiferroquadrupolar ordered state of $\mathrm{CeB}_{6}$. Phys. Rev. Lett. 86, 1578-1581 (2001).

26. Sera, M. \& Kobayashi, S. Magnetic properties of the 4 sublattice model for the antiferro (AF) quadrupolar order dominated by the $\mathrm{AF}$ octupolar and $\mathrm{AF}$ exchange interactions -a simple model for $\mathrm{CeB}_{6}-$. J. Phys. Soc. Jpn. 68, 1664-1678 (1999).

27. Mannix, D., Tanaka, Y., Carbone, D., Bernhoeft, N. \& Kunii, S. Order parameter segregation in $\mathrm{Ce}_{0.7} \mathrm{La}_{0.3} \mathrm{~B}_{6}: 4 f$ octopole and $5 d$ dipole magnetic order. Phys. Rev. Lett. 95, 117206 (2005).

28. Kuwahara, K. et al. Detection of neutron scattering from phase IV of $\mathrm{Ce}_{0.7} \mathrm{La}_{0.3} \mathrm{~B}_{6}$ : a confirmation of the octupole order. J. Phys. Soc. Jpn. 76, 093402 (2007).

29. Matsumura, T. et al. Antiferroquadrupole order and magnetic field induced octupole in $\mathrm{CeB}_{6}$. Phys. Rev. B 85, 174417 (2012).

30. Matsumura, $T$. et al. Evidence for hidden quadrupolar fluctuations behind the octupole order in $\mathrm{Ce}_{0.7} \mathrm{La}_{0.3} \mathrm{~B}_{6}$ from resonant $x$-ray diffraction in magnetic fields. Phys. Rev. B 89, 014422 (2014).
31. Ohkawa, J. F. Ordered state in periodic Anderson hamiltonian with orbital degeneracy and with large coulomb correlation. J. Phys. Soc. Jpn. 52, 3897-3906 (1983).

32. Ohkawa, J. F. Orbital antiferromagnetism in $\mathrm{CeB}_{6}$. J. Phys. Soc. Jpn. 54, 3909-3914 (1985).

33. Shiina, R., Shiba, H. \& Thalmeier, P. Magnetic-field effects on quadrupolar ordering in a $\Gamma_{8}$-quartet system $\mathrm{CeB}_{6}$. J. Phys. Soc. Jpn. 66, 1741-1755 (1997).

34. Schell, G., Winter, H., Rietschel, H. \& Gompf, F. Electronic structure and superconductivity in metal hexaborides. Phys. Rev. B 25, 1589-1599 (1982).

35. Cameron, A. S., Friemel, G. \& Inosov, D. S. Multipolar phases and magnetically hidden order: review of the heavy-fermion compound $\mathrm{Ce}_{1-x} \mathrm{La}_{x} \mathrm{~B}_{6}$. Rep. Prog. Phys. 79, 066502 (2016).

36. Friemel, G. et al. Magnetic field and doping dependence of low-energy spin fluctuations in the antiferroquadrupolar compound $\mathrm{Ce}_{1-x} \mathrm{La}_{x} \mathrm{~B}_{6}$. Phys. Rev. B 92, 014410 (2015).

37. Friemel, G. 'Itinerant spin dynamics in iron-based superconductors and ceriumbased heavy-fermion antiferromagnets' Ph. D. Thesis, Fakultät Mathematik und Physik, Universität Stuttgart (2014).

38. Zirngiebl, E. et al. Crystal-field excitations in $\mathrm{CeB}_{6}$ studied by Raman and neutron spectroscopy. Phys. Rev. B 30, 4052-4054 (1984).

39. Nakamura, S., Goto, T., Suzuki, O., Kunii, S. \& Sakatsume, S. Screening effect on the multipole moments in the Kondo system $\mathrm{Ce}_{x} \mathrm{La}_{1-x} \mathrm{~B}_{6}$. Phys. Rev. $B$ 61, 15203-15212 (2000).

40. Portnichenko, P. Y. et al. Incommensurate short-range multipolar order parameter of phase II in $\mathrm{Ce}_{3} \mathrm{Pd}_{20} \mathrm{Si}_{6}$. Phys. Rev. B 94, 245132 (2016).

41. Schotte, K. D. \& Schotte, U. Interpretation of Kondo experiments in a magnetic field. Phys. Lett. 55A, 38-40 (1975).

42. Desgranges, H. U. \& Schotte, K. D. Specific heat of the Kondo model. Phys. Lett. 91A, 240-242 (1982).

43. Nakamura, S., Goto, T. \& Kunii, S. Magnetic phase diagrams of the dense Kondo compounds $\mathrm{CeB}_{6}$ and $\mathrm{Ce}_{0.5} \mathrm{La}_{0.5} \mathrm{~B}_{6}$. J. Phys. Soc. Jpn. 64, 3941-3945 (1995).

44. Akatsu, M. et al. Trigonal lattice distortion and ferro-quadrupole ordering in phase IV of $\mathrm{Ce}_{x} \mathrm{La}_{1-x} \mathrm{~B}_{6}(x=0.75$ and 0.70). J. Phys. Soc. Jpn. 72, 205-208 (2003).

45. Nakamura, $S$. et al. Unusual evolution of the conduction-electron state in $\mathrm{Ce}_{x} \mathrm{La}_{1-x} \mathrm{~B}_{6}$ from non-Fermi liquid to Fermi liquid. Phys. Rev. Lett. 97, 237204 (2006).

46. Jiao, L. et al. Additional energy scale in $\mathrm{SmB}_{6}$ at low-temperature. Nat. Commun. 7, 13762 (2016).

47. Jang, $H$. et al. Intense low-energy ferromagnetic fluctuations in the antiferromagnetic heavy-fermion metal $\mathrm{CeB}_{6}$. Nat. Mater. 13, 682-687 (2014).

48. Friemel, G. et al. Resonant magnetic exciton mode in the heavy-fermion antiferromagnet $\mathrm{CeB}_{6}$. Nat. Commun. 3, 830 (2012).

49. Wilhelm, H., Lühmann, T., Rus, T. \& Steglich, F. A compensated heat-pulse calorimeter for low temperatures. Rev. Sci. Instrum. 75, 2700-2705 (2004).

50. Schneidewind, A. \& Čermák, P. PANDA: cold three axes spectrometer. J. Largescale Res. Facil. 1, A12 (2015).

Open Access This article is licensed under a Creative Commons Attribution 4.0 International License, which permits use, sharing, adaptation, distribution and reproduction in any medium or format, as long as you give appropriate credit to the original author(s) and the source, provide a link to the Creative Commons license, and indicate if changes were made. The images or other third party material in this article are included in the article's Creative Commons license, unless indicated otherwise in a credit line to the material. If material is not included in the article's Creative Commons license and your intended use is not permitted by statutory regulation or exceeds the permitted use, you will need to obtain permission directly from the copyright holder. To view a copy of this license, visit http://creativecommons. org/licenses/by/4.0/.

(c) The Author(s) 2017 